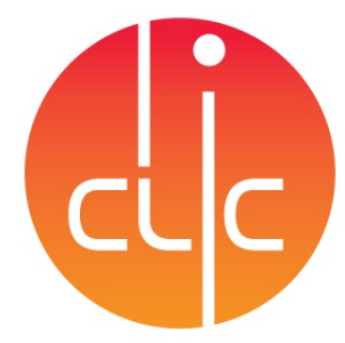

CLIC - Note - 985

\title{
BRILLIANT POSITRON SOURCES FOR CLIC AND OTHER COLLIDER PROJECTS
}

\author{
Louis Rinolfi ${ }^{\mathrm{a}}$, Robert Chehab ${ }^{\mathrm{b}}$, Olivier Dadoun ${ }^{\mathrm{c}}$, Takuya Kamitani $^{\mathrm{d}}$, Vladimir Strakhovenko ${ }^{\mathrm{e}}$, \\ Alessandro Variola ${ }^{\mathrm{C}}$ \\ ${ }^{a}$ CERN, Geneva, Switzerland \\ ${ }^{\mathrm{b}}$ IPNL, Lyon, France \\ ${ }^{\mathrm{c}}$ LAL, Orsay, France \\ ${ }^{\mathrm{d}}$ KEK, Tsukuba, Japan \\ e BINP, Novosibirsk, Russia
}

\begin{abstract}
The CLIC (Compact Linear Collider), as future linear collider, requires an intense positron source. A brief history is given up to the present baseline configuration which assumes unpolarized beams. A conventional scheme, with a single tungsten target as source of $\mathrm{e}^{-} \mathrm{e}^{+}$pairs, has been studied several years ago. But, in order to reduce the beam energy deposition on the $\mathrm{e}^{+}$target converter, a double-target system has been studied and proposed as baseline for CLIC. With this "hybrid target', the positron production scheme is based on the channeling process. A $5 \mathrm{GeV}$ electron beam impinges on a thin crystal tungsten target aligned along its $<111>$ axis, enhancing the photon production by channeling radiation. A large number of photons are sent to a thick amorphous tungsten target, generating large number of $\mathrm{e}^{-} \mathrm{e}^{+}$pairs, while the charged particles are bent away, reducing the deposited energy and the PEDD (Peak Energy Deposition Density). The targets parameters are optimized for the positron production. Polarized positron beams are an option for CLIC, which needs R\&D. Some brilliant positron sources are briefly reviewed.
\end{abstract}

Presented at Channeling workshop, 23-28 September 2012, Alghero, Italy

Geneva, Switzerland

December 2012 


\title{
Brilliant positron sources for CLIC and other collider projects
}

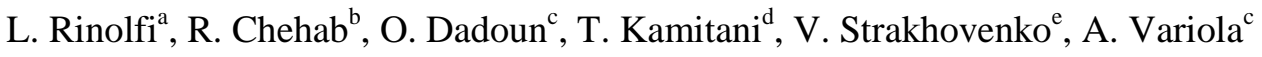 \\ ${ }^{a}$ CERN, Geneva (Switzerland) \\ ${ }^{\mathrm{b}}$ IPNL, Lyon (France) \\ ${ }^{\mathrm{c}}$ LAL, Orsay (France) \\ ${ }^{\mathrm{d}}$ KEK, (Japan) \\ ${ }^{\mathrm{e}}$ BINP, Novosibirsk, Russia
}

\section{Introduction}

A very brief history of the CLIC (Compact Linear Collider) study is given in the next paragraph. A possible design of a multi-TeV linear collider is described in [1]. The CLIC study has been presented in major conferences (PAC, EPAC, IPAC, Linear Accelerator,...), in the 3 CLIC workshops (2007, 2008, 2009) and in all Linear Colliders workshops (LCWS), since the years 1990's. The study of the CLIC positron source started in 1994, based on conventional target. Ten years later, collaboration started with the ILC (International Linear Collider), although it considers the undulator as the baseline for positron source.

At the LCWS'08 workshop in Chicago, a new ILC/CLIC common working group was created as " $\mathrm{e}^{+}$ sources" [2]. The present beam parameters and the corresponding layout for the main beam injector complex are given. The studies based on conventional positron sources are described first, followed by the studies optimizing hybrid targets configuration where channeling process occurs. The various approaches to generate polarized $\mathrm{e}^{+}$beam for CLIC are presented. Finally, projects involving other brilliant positron sources are mentioned.

\section{A very brief CLIC history}

In 1985, when the study started, the acronym CLIC stood for “CERN Linear Collider” and the first CLIC Note was published in August [3].

Ten years later, in 1995, it becomes obvious that such linear collider would be built only within an international collaboration and the acronym of CLIC became "Compact Linear Collider". At this time, six linear collider projects were studied around the world: TESLA, SBLC (S-Band Linear Collider), JLC (Joint Linear Collider), NLC (Next Linear Collider), VLEPP (Super high Energy Colliding Electron-Positron Beam Facility) and CLIC (Compact Linear Collider).

In 2004, an International Technology Recommendation Panel selects the Superconducting RF technology (TESLA based) versus room temperature copper structures (JLC/NLC based). The ILC (International Linear Collider) was born, based on RF frequency at $1.3 \mathrm{GHz}$ for the TeV scale while CLIC study continues with a RF frequency at $30 \mathrm{GHz}$ for the multi-TeV scale.

In 2007, major changes occurred in the CLIC study: The RF frequency of $30 \mathrm{GHz}$ was reduced down to $12 \mathrm{GHz}$ and the accelerating gradient was reduced from $150 \mathrm{MV} / \mathrm{m}$ down to $100 \mathrm{MV} / \mathrm{m}$.

In 2008, a successful test of a CLIC structure is obtained at $12 \mathrm{GHz}$ within an international collaboration; this structure was designed at CERN, built at KEK and tested at SLAC. The same year, a new ILC/CLIC common working group was created as " $\mathrm{e}^{+}$sources" [2].

In July 2012, there was an observation at LHC of particle consistent with long-sought Higgs boson. Today, the CDR (Conceptual Design Report) is published [4] with more than 950 CLIC Notes. The CLIC web page [5] provides the up-to-date data and references to the CLIC Notes. 


\section{Overview of the CLIC and its main beam injector complex}

The nominal CLIC parameters are given in the Table 1.

Table 1: CLIC Main Beam parameters [6]

\begin{tabular}{|l|c|c|}
\hline Center-of-mass energy & $0.5 \mathrm{TeV}$ & $3 \mathrm{TeV}$ \\
\hline Peak Luminosity & $2.3 \times 10^{34} \mathrm{~cm}^{-2} \mathrm{~s}^{-1}$ & $5.9 \times 10^{34} \mathrm{~cm}^{-2} \mathrm{~s}^{-1}$ \\
\hline Peak Luminosity (in 1\% of energy) & $1.4 \times 10^{34} \mathrm{~cm}^{-2} \mathrm{~s}^{-1}$ & $2 \times 10^{34} \mathrm{~cm}^{-2} \mathrm{~s}^{-1}$ \\
\hline Repetition rate & $50 \mathrm{~Hz}$ & $50 \mathrm{~Hz}$ \\
\hline Loaded accelerating gradient & $80 \mathrm{MV} / \mathrm{m}$ & $100 \mathrm{MV} / \mathrm{m}$ \\
\hline Main Linac RF frequency & $12 \mathrm{GHz}$ & $12 \mathrm{GHz}$ \\
\hline Bunch charge at IP & $6.8 \times 10^{9}$ & $3.72 \times 10^{9}$ \\
\hline Number of bunches & 354 & 312 \\
\hline Bunch spacing & $0.5 \mathrm{~ns}$ & $0.5 \mathrm{~ns}$ \\
\hline Beam pulse duration & $177 \mathrm{~ns}$ & $156 \mathrm{~ns}$ \\
\hline Beam power per beam & $4.9 \mathrm{MW}$ & $14 \mathrm{MW}$ \\
\hline Horizontal / Vertical emittances & $2400 / 25 \mathrm{~nm}$ & $660 / 20 \mathrm{~nm}$ \\
\hline Horizontal / Vertical beam size at IP before pinch & $202 / 2.3 \mathrm{~nm}$ & $40 / 1 \mathrm{~nm}$ \\
\hline Total site length & $13 \mathrm{~km}$ & $48.3 \mathrm{~km}$ \\
\hline Total power consumption & $130 \mathrm{MW}$ & $415 \mathrm{MW}$ \\
\hline
\end{tabular}

Figure 1 shows the general CLIC layout.

On the top of the Figure, the Drive Beam complex generates the $12 \mathrm{GHz}$ RF power. In the middle, the Main Linacs allow the acceleration up to $3 \mathrm{TeV}$ at IP. On the bottom, a part of the Main Beam complex is shown where the main colliding beams are produced in conventional electron and positron sources. The present baseline design foresees $80 \%$ polarisation of the electrons at collision, no polarized positron but it is compatible with the addition of a polarised positron source.

The constraints for the design study of the brilliant positron source are derived from the parameters requested at the IP.

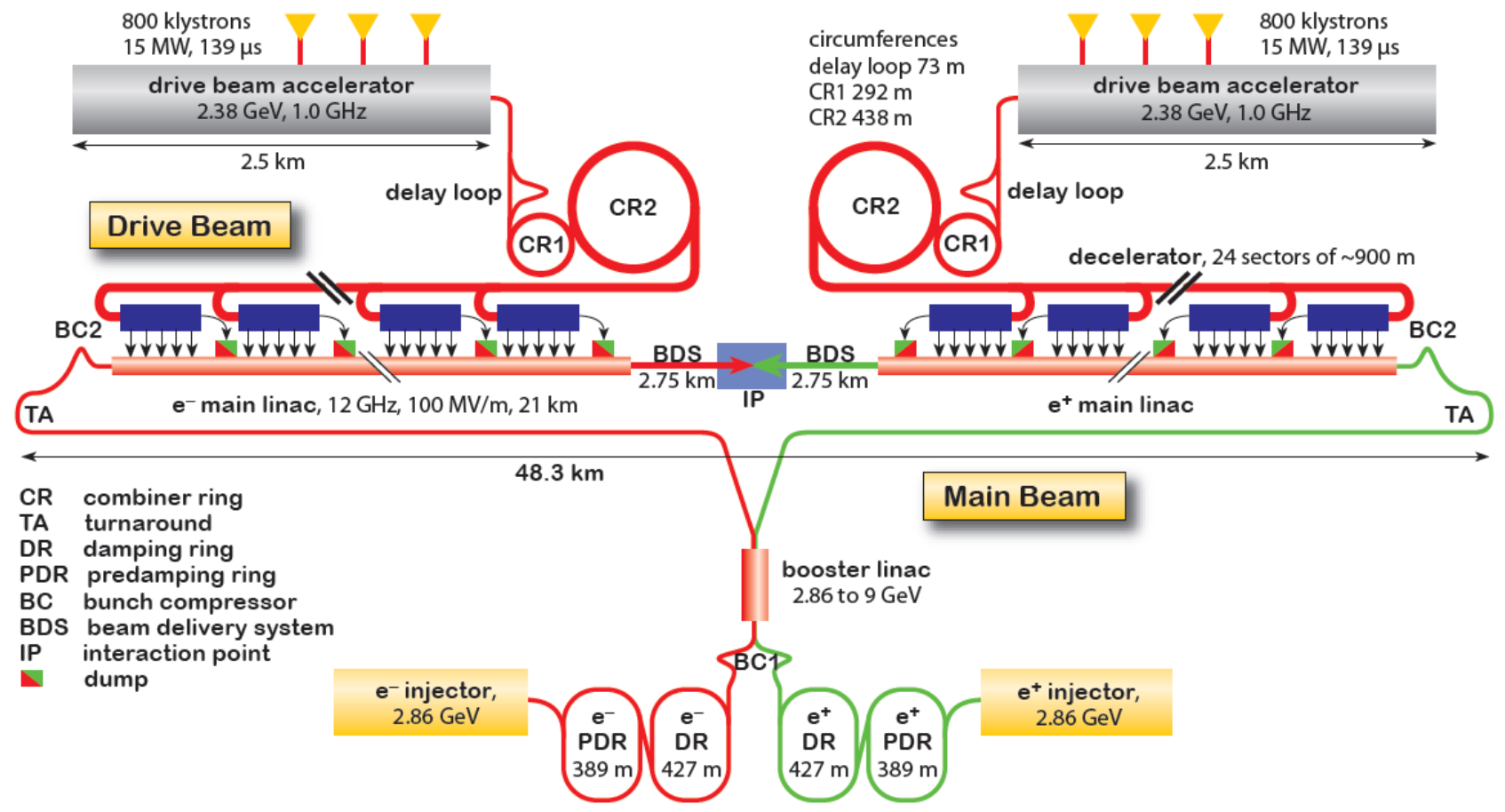

Figure 1: CLIC general layout for $3 \mathrm{TeV}$ 
Figure 2 shows the Main Beam Injector complex for the baseline configuration.

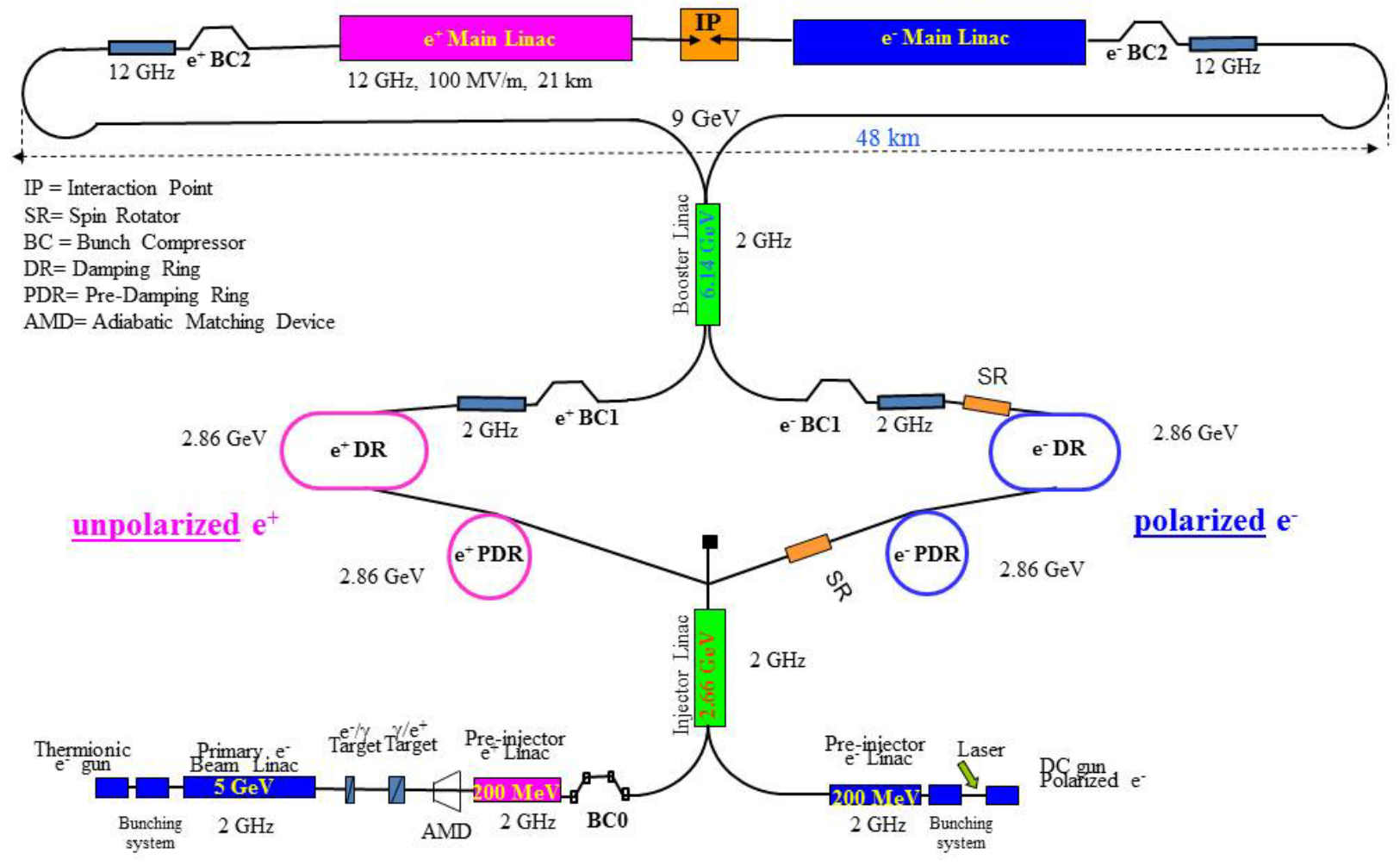

Figure 2: CLIC Main Beam Injector Complex

i) The electron generation: One photo-cathode with a DC gun generates beam at $120 \mathrm{keV}$ [7] with a charge of $6 \times 10^{9} \mathrm{e}^{-} /$microbunch. A sophisticated laser system illuminates the photo-cathode. One bunching system and sub-harmonic bunchers provides bunched beam at $10 \mathrm{MeV}$. One pre-injector linac accelerates the $\mathrm{e}^{-}$ beam at $200 \mathrm{MeV}$.

ii) The positron generation: A thermionic gun with a bunching system followed by a Primary Beam Linac accelerates $\mathrm{e}^{-}$beam at $5 \mathrm{GeV}$ onto the $\mathrm{e}^{+}$target. The positron source itself is composed of hybrid targets: one thin crystal target, followed by one amorphous target and an Adiabatic Matching Device (AMD). A positron Pre-injector Linac accelerates $\mathrm{e}^{+}$(and secondary e $\mathrm{e}^{-}$) up to $200 \mathrm{MeV}$.

iii) A unique Injector linac: This linac accelerates alternately the train of electrons and the train of positrons. The Injector linac [8] accelerates beams at $2.86 \mathrm{GeV}$. At the end of the Linac, a DC dipole magnet separates the $\mathrm{e}^{-}$beam and the $\mathrm{e}^{+}$beam. When this dipole is switched off, the beam is sent towards a dump where beam instrumentation is implemented.

iv) The generation of small beam emittances: A pre-damping ring and a damping ring for $\mathrm{e}^{-}$are used to reduce the transverse beam emittances to the requested values. Similarly a pre-damping ring [9] and a damping ring for $\mathrm{e}^{+}$are implemented to reduce the transverse beam normalized emittances from $10^{4} \mathrm{~mm} . \mathrm{mrad}$ down to $400 \mathrm{~nm} \cdot \operatorname{rad}(\mathrm{H})$ and $5 \mathrm{~nm} \cdot \operatorname{rad}(\mathrm{V})$.

v) The transport [10] of the tiny emittances through the RTML (Ring To Main Linac) line: A first stage of bunch compressor works at $2 \mathrm{GHz}$ and $2.86 \mathrm{GeV}$ (one for each beam). A unique Booster linac accelerates alternately both beams up to $9 \mathrm{GeV}$. A long transfer line for $\mathrm{e}^{-}$and a long transfer line for $\mathrm{e}^{+}$are foreseen. A second stage of bunch compressor at $12 \mathrm{GHz}$ and $9 \mathrm{GeV}$ just upstream of the injection into the Main Linac (one for each beam) is implemented. 


\section{Conventional $\mathrm{e}^{+}$production scheme with single target}

A first study related to the main beams injector complex is reported in [11]. The $\mathrm{e}^{+}$production is based on standard technology developed at SLC and using a single target. The number of bunches is 4 but the repetition rate is $1700 \mathrm{~Hz}$.

In 1997, preliminary simulations [12] were performed for an $\mathrm{e}^{+}$target, similar to the SLC target, $\mathrm{W}_{75} \mathrm{Re}_{25}$. With the beam parameters used at this time, $8 \times 10^{10} \mathrm{e}^{-} /$pulse at $1.98 \mathrm{GeV}$ and $511 \mathrm{~Hz}$ repetition rate, the temperature increase is $630{ }^{\circ} \mathrm{C}$ at the exit of the target. Figure 3 shows the geometry of the target.

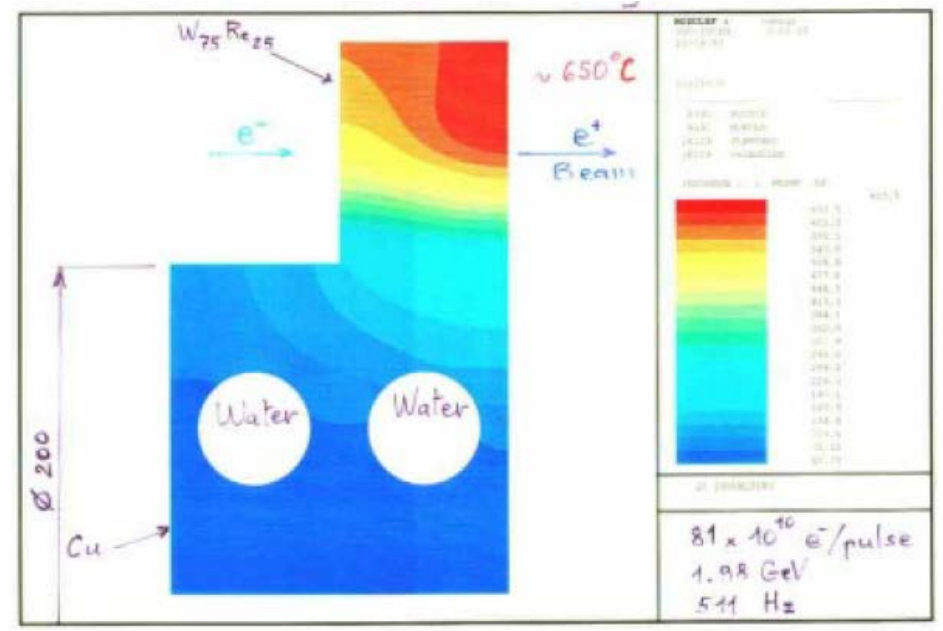

Figure 3: CLIC $\mathrm{e}^{+}$target

For the LCWS'97, studies and proposals concerning the positron source and other components (RF gun, damping rings, bunch compressors, ...) of the Main Beam Injector complex were reported in [13].

In 2000, detailed studies and simulations [14] were performed according to the new CLIC parameters. Simulations used EGS4 for the energy deposition inside the target. A new tracking simulation program SOLEIL (SOLEnoid focusing sImuLator) was developed. It is based on the code which was implemented for the design of the positron generator of the KEKB factory injector linac. The trajectories of the particles are traced by step-by-step numerical integration of the relativistic equations of motion with the $4^{\text {th }}$ order Runge-Kutta method. The simulation results have been compared to analytical formula [15] and showed rather good agreement. Figure 4 gives a layout of the CLIC $\mathrm{e}^{+}$source up to the exit of the capture section at $200 \mathrm{MeV}$ with the corresponding characteristics. The simulations results related to these parameters are reported in [16].

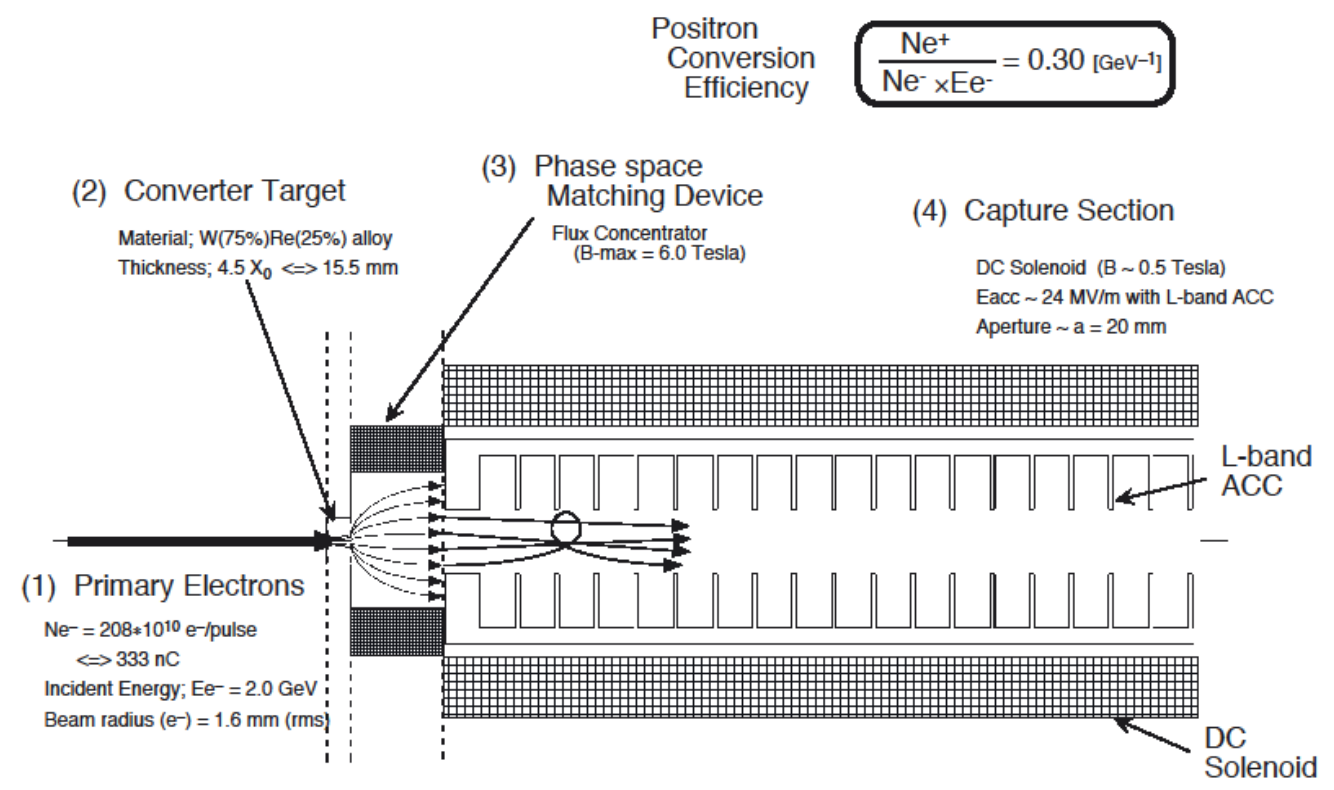

Figure 4: Layout of the CLIC $\mathrm{e}^{+}$source with a single target 
With the new CLIC parameters, it becomes challenging to use a single target as $\mathrm{e}^{+}$source. The issues are related to the beam energy deposition and the shock waves. Simulations have been performed with FLUKA code [17] and GEANT4 and confirm the risks of breakdowns. Figure 5 shows a plot obtained with FLUKA code showing a peak of $1.4 \mathrm{MeV} / \mathrm{e}^{-}$which leads to a PEDD close to the breakdown limit (35 J/g for the tungsten). The conditions are identical to those described in [14].

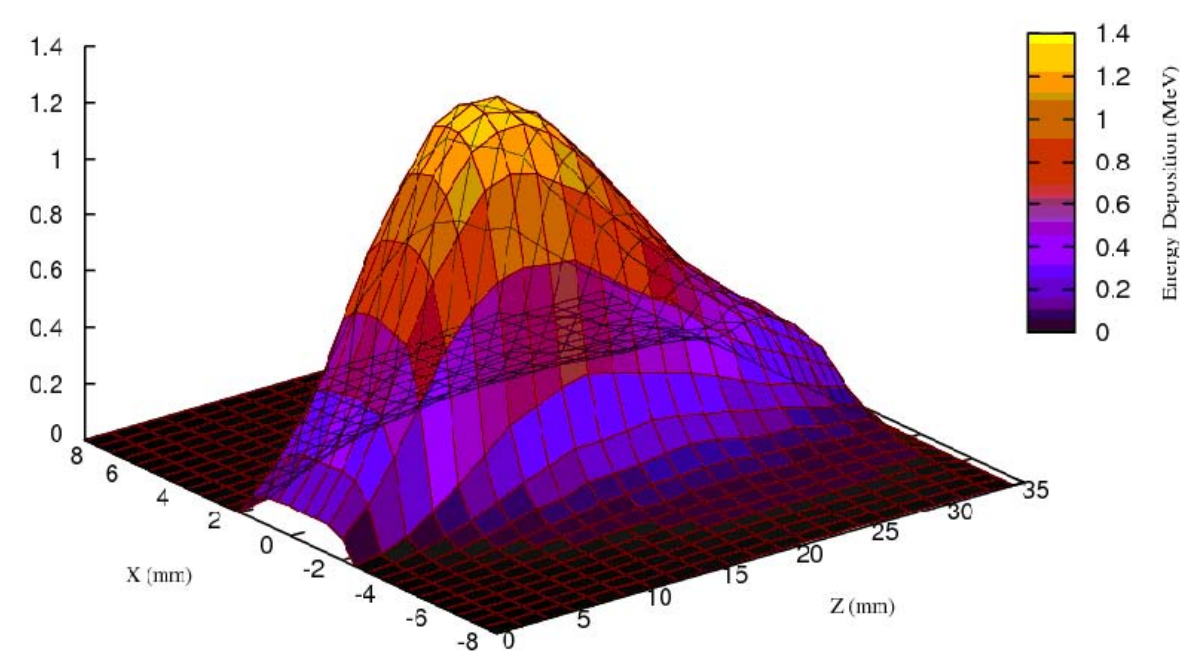

Figure 5: Energy deposition in the CLIC target from FLUKA code

Since several projects requiring a brilliant $\mathrm{e}^{+}$sources are under study, it is interesting to compare what is presently requested with the SLC $\mathrm{e}^{+}$source (which was the only one built for a linear collider). Table 2 shows the $\mathrm{e}^{+}$flux for the SLC (Stanford), CLIC (2 energies: $3 \mathrm{TeV}$ and $0.5 \mathrm{TeV}$ ), ILC (Reference Design Report) and LHeC (pulsed option and CW option with an ERL). For CLIC (3 TeV), a factor 20 is needed compared to SLC.

Table 2: Positron flux for different studies compared to the SLC

\begin{tabular}{|c|c|c|c|c|c|c|}
\hline & SLC & $\begin{array}{c}\text { CLIC } \\
(3 \mathrm{TeV})\end{array}$ & $\begin{array}{c}C L I C \\
(0.5 \mathrm{TeV})\end{array}$ & $\begin{array}{c}\text { ILC } \\
\text { (RDR) }\end{array}$ & $\begin{array}{l}\text { LHeC } \\
\text { (pulsed) }\end{array}$ & $\begin{array}{l}\text { LHeC } \\
\text { ERL }\end{array}$ \\
\hline Energy & $1.19 \mathrm{GeV}$ & $2.86 \mathrm{GeV}$ & $2.86 \mathrm{GeV}$ & $5 \mathrm{GeV}$ & $140 \mathrm{GeV}$ & $60 \mathrm{GeV}$ \\
\hline $\begin{array}{l}e^{+} / \text {bunch } \\
\text { (at IP) }\end{array}$ & $40 \times 10^{9}$ & $3.7 \times 10^{9}$ & $7.4 \times 10^{9}$ & $20 \times 10^{9}$ & $1.6 \times 10^{9}$ & $2 \times 10^{9}$ \\
\hline $\begin{array}{l}\mathrm{e}^{+} / \text {bunch } \\
\text { (aft. capture) }\end{array}$ & $50 \times 10^{9}$ & $7 \times 10^{9}$ & $14 \times 10^{9}$ & $30 \times 10^{9}$ & $1.8 \times 10^{9}$ & $2.2 \times 10^{9}$ \\
\hline $\begin{array}{l}\text { Bunches / } \\
\text { macropulse }\end{array}$ & 1 & 312 & 354 & 2625 & 100000 & NA \\
\hline $\begin{array}{l}\text { Rep. Rate } \\
(\mathrm{Hz})\end{array}$ & 120 & 50 & 50 & 5 & 10 & $C W$ \\
\hline Bunches / s & 120 & 15600 & 17700 & 13125 & $10^{6}$ & $20 \times 10^{6}$ \\
\hline $\begin{array}{c}\mathrm{e}^{+} / \text {second } \\
\times 10^{14}\end{array}$ & 0.06 & 1.1 & 2.5 & 3.9 & 18 & 440 \\
\hline
\end{tabular}

For CLIC $(0.5 \mathrm{TeV})$, the charge is double and therefore the $\mathrm{e}^{+}$flux requires a factor greater than 40 compared to SLC, which is certainly a big challenge. 


\section{The hybrid target based on channeling process as $\mathrm{e}^{+}$source}

The idea to use channeling effects to enhance the $\mathrm{e}^{+}$positron sources, for linear colliders, emerged in 1989 [18]. However this relies on the long term resistance of the crystal to radiation damages. Such damages have been tested on a $0.3 \mathrm{~mm}$ thick tungsten monocrystal exposed during 6 months to the $30 \mathrm{GeV}$ incident electron beam of the SLAC Linear Collider (SLC). The crystal was placed in the converter region, orientated in a random direction and received an integrated flux of $2.10^{18} \mathrm{e}^{-/} / \mathrm{mm}^{2}$. The results [19] show that no damages occurred during these irradiations.

Later on, an experiment operated at CERN (WA 103) showed significant enhancements in positron production between $\mathrm{W}$ crystal and amorphous targets both of the same thickness: a factor 2, for an $8 \mathrm{~mm}$ target and a factor 4 , for $4 \mathrm{~mm}$ target [20, 21].

\subsection{Simulations to optimize the $e^{+}$production for the present CLIC parameters}

According to the most recent CLIC beam parameters, the $\mathrm{e}^{+}$production is optimized using a hybrid targets configuration. This concept was proposed in [22] and represented in Figure 6. With a tungsten crystal oriented on its <111> axis, one obtains an intense, relatively low energy photon beam due mainly to channeling radiation. Those photons are then impinging on an amorphous tungsten target producing positrons by $\mathrm{e}^{+} \mathrm{e}^{-}$pair creation. The optimization of the positron yield and the peak energy deposition density in the amorphous target are studied [23] according to the distance between the crystal and the amorphous targets, the primary electron energy and the amorphous target thickness.

Although the target is crucial for a brilliant $\mathrm{e}^{+}$source, the optics downstream also plays a crucial role for the performance of the positrons. Therefore simulations have been performed from the exit of the amorphous target up to the entrance of the Pre-Damping Ring. Downstream the amorphous target, there is an Adiabatic Matching Device (AMD) followed by a Pre-Injector Linac accelerating the $\mathrm{e}^{+}$beam up to $200 \mathrm{MeV}$. No beam instrumentation could be installed before $200 \mathrm{MeV}$ and one should rely on simulations only $[24,25]$. The characteristics of the AMD and the Pre-Injector Linac are given in the same references.

Following intense studies, it is deduced that the positron yield obtained at the exit of the Pre-Injector Linac fulfills the CLIC requirements, using a hybrid targets scheme with parameters presented in [26].

\subsection{Targets}

The conventional positron source is based on an electromagnetic shower created by electrons impinging on high-Z material target. For the CLIC baseline, channeling process occurs inside the thin crystal target which receives high-energy electrons, producing $\mathrm{e}^{+} / \mathrm{e}^{-}$pairs and photons. The latter are sent to the thick amorphous target producing $\mathrm{e}^{+} \mathrm{e}^{-}$pairs while the electrons and positrons are swept around between the crystal target and the thick target. As already mentioned, an important issue is the beam deposition power inside the amorphous target. The failure threshold for $\mathrm{W}$ target was measured, at SLAC, and found to be around $35 \mathrm{~J} / \mathrm{g}$. For the amorphous CLIC target, and based on results obtained in [23], the values of the present optimized configuration are given in Figure 6.

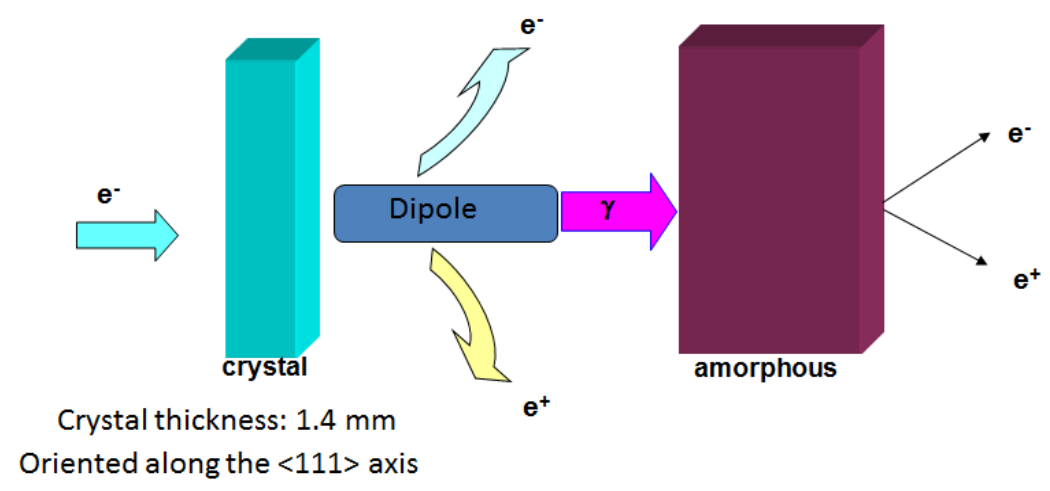

Distance (crystal-amorphous) $\mathrm{d}=2 \mathrm{~m}$

Amorphous thickness $e=10 \mathrm{~mm}$

Figure 6: Optimized CLIC hybrid targets configuration 
Table 3 gives a summary for the CLIC hybrid targets. The beam power on the crystal target is $94 \mathrm{~kW}$. With a repetition rate of $50 \mathrm{~Hz}$, the deposited power in the crystal target is $0.2 \mathrm{~kW}$ while the deposited power in the amorphous target is $7.5 \mathrm{~kW}$.

Table 3: CLIC e ${ }^{+}$targets

\begin{tabular}{|l|c|c|c|}
\hline Target & & Crystal & Amorph. \\
\hline Material & & $\mathrm{W}$ & $\mathrm{W}$ \\
\hline Length & $\mathrm{mm}$ & 1.4 & 10 \\
\hline Radiation lengths & $\chi_{0}$ & 0.4 & 2.9 \\
\hline Beam power deposited & $\mathrm{kW}$ & 0.2 & 7.5 \\
\hline Deposited P / Beam Power & $\%$ & 0.2 & 8 \\
\hline Energy lost per volume & $10^{9} \mathrm{GeV} / \mathrm{mm}^{3}$ & 0.8 & 1.9 \\
\hline Peak Energy Deposition Density (PEDD) & $\mathrm{J} / \mathrm{g}$ & 7 & 15 \\
\hline
\end{tabular}

Preliminary and encouraging experimental results, using hybrid targets, are obtained at KEK [27]. Figure 7 shows the $\mathrm{e}^{+}$yield measured downstream the amorphous target versus the thickness of the amorphous target. The blue color is for a conventional amorphous target. The red is for hybrid targets using channeling. The green is for hybrid target but the crystal target is rotated and behaves as amorphous. Experimental points are compared to simulations for energy of $20 \mathrm{MeV}$.

The limited acceptance of the experimental set-up on the KEKB Linac and the expected larger divergence of the positrons generated in the hybrid target, could explain the better $\mathrm{e}^{+}$yield (above $\sim 8 \mathrm{~mm}$ ) for the conventional case. Further verifications and checks are on-going at KEK.

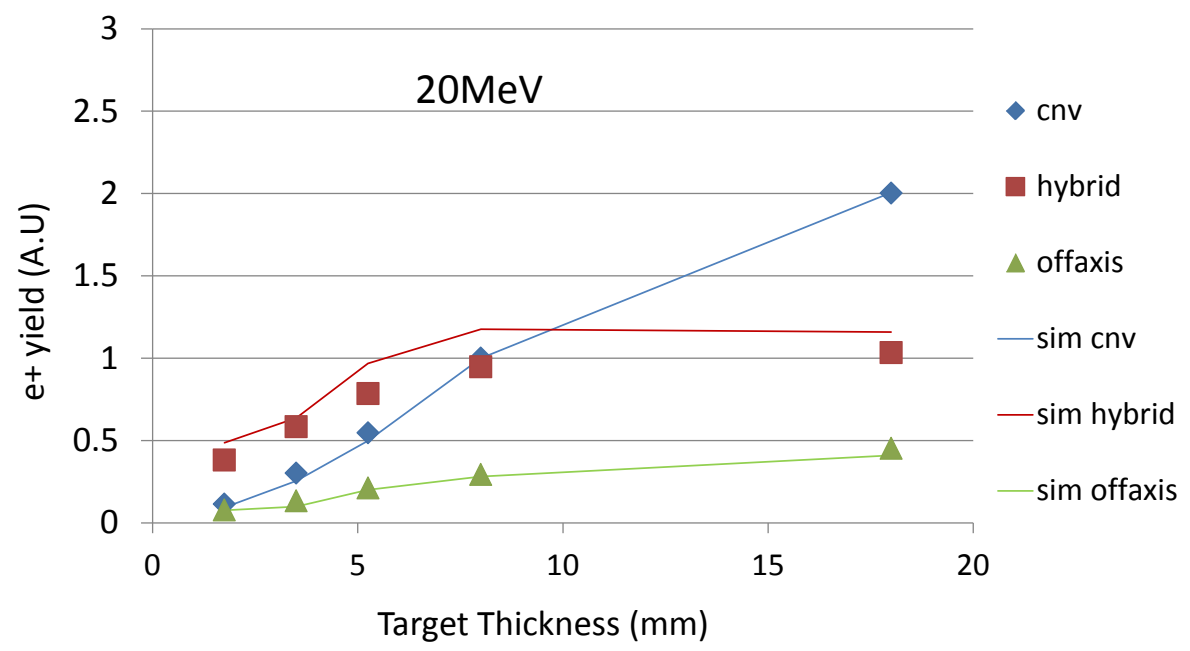

Figure 7: Experimental results at KEKB linac

\section{The polarized $\mathrm{e}^{+}$for CLIC}

The source is based on a positron production scheme in which polarized photons are produced by a Compton process [28]. One option is the Compton backscattering, where an electron beam of $\sim 1 \mathrm{GeV}$ interacts with circularly-polarized photons in an optical resonator [29]. The resulting circularly-polarized gamma photons are sent on to an amorphous target, producing pairs of longitudinally polarized electrons and positrons [30]. Figure 8 shows a possible configuration. 


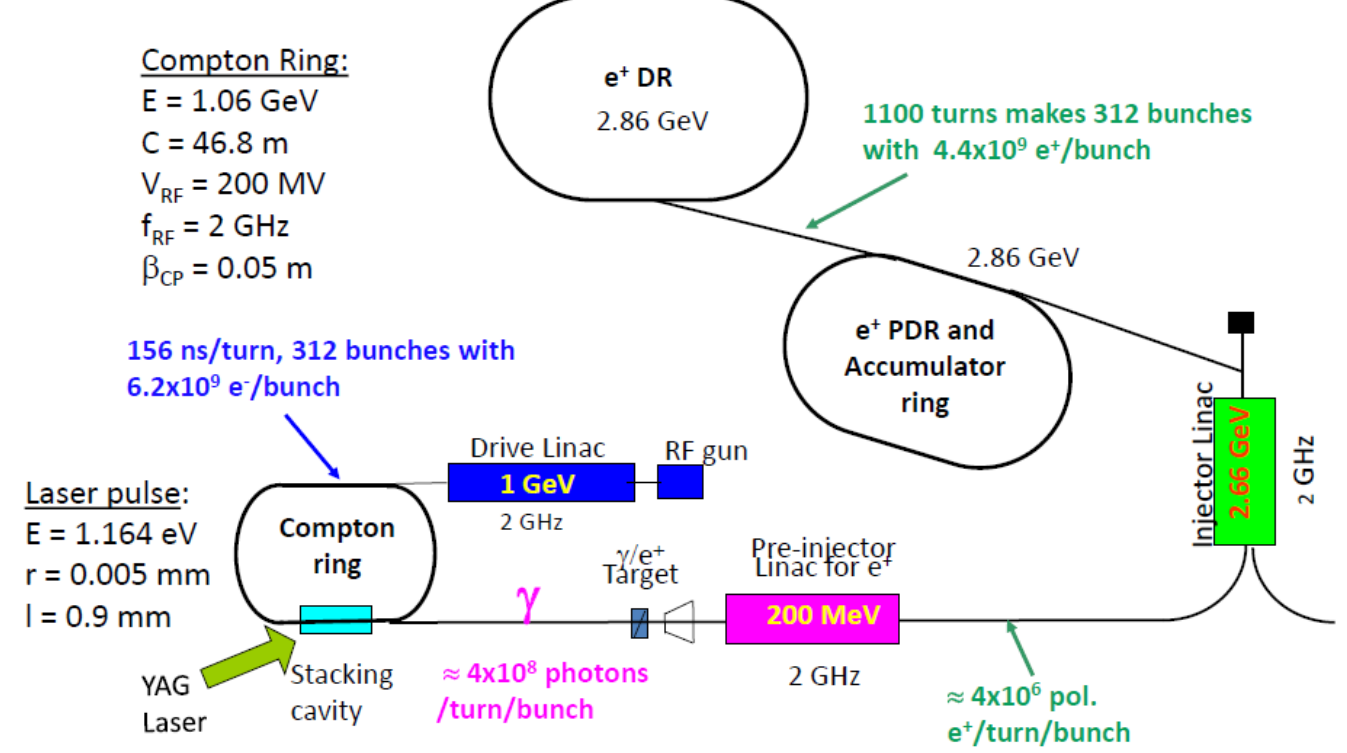

Figure 8: Compton ring as source of polarized $\mathrm{e}^{+}$for CLIC

For CLIC, the photon flux coming out from a "Compton ring" is not sufficient to obtain the requested charge. Therefore a stacking process is required somewhere (Pre-Damping Ring, Stacking Ring) [31, 32]. Another option is to use a Compton Energy Recovery Linac (ERL) where a quasicontinual stacking could be achieved [33, 34]. A third option is to use a "Compton Linac" which would not require stacking [35].

A study where an undulator is implemented into the CLIC tunnel has been made. Based on the simulation results, the polarization of $60 \%$ can be achieved by using a $127 \mathrm{~m}$ long undulator with $\mathrm{K}=0.9$, $\lambda_{\mathrm{u}}=1.15 \mathrm{~cm}$ and a photon collimator with an iris of $0.7 \mathrm{~mm}$. The Ti target $\left(0.4 \chi_{0}\right.$ length) is located $400 \mathrm{~m}$ away from the end of the undulator [36, 37].

\section{Other brilliant $\mathbf{e}^{+}$sources}

\subsection{ILC $\mathrm{e}^{+}$sources}

The baseline for the ILC positron source is using the circularly polarized photons generated by a high energy electron beam $(\mathrm{E}>150 \mathrm{GeV})$ in a helical undulator to produce longitudinally polarized positrons in a thin amorphous target. Collimation of the photons coming out from the undulator is essential, so the conversion target is put at a large distance from the undulator, at least a distance equal to the length of the undulator. Figure 9a shows a general layout of the ILC and Figure 9b shows a layout of the positron source which is described in the RDR [38]. Due to the collimation, the photon spot is rather small and hence the lateral dimensions of the shower in the converter. That leads to important energy deposition densities. Henceforth, a rotating target with high velocity $(100 \mathrm{~m} / \mathrm{s}$, tangential) is considered [39]. A proof of principle, at a reduced power, was successfully operated at SLAC (E-166) [40].

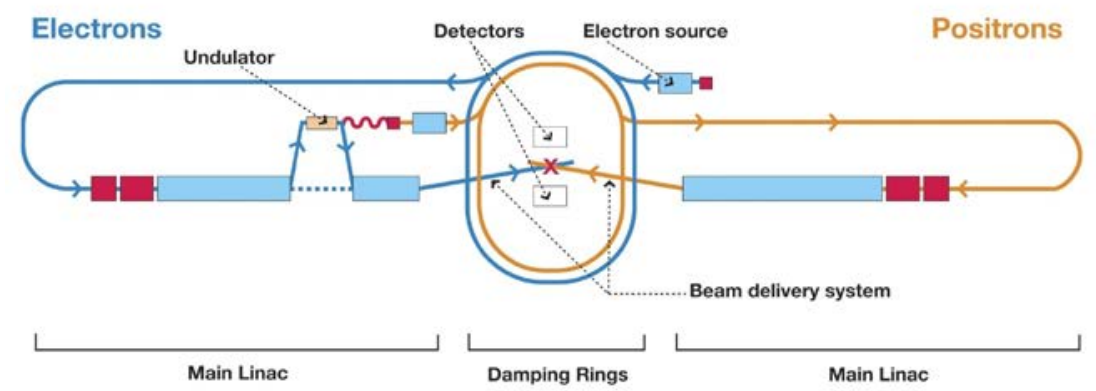

Figure 9a: ILC general layout 


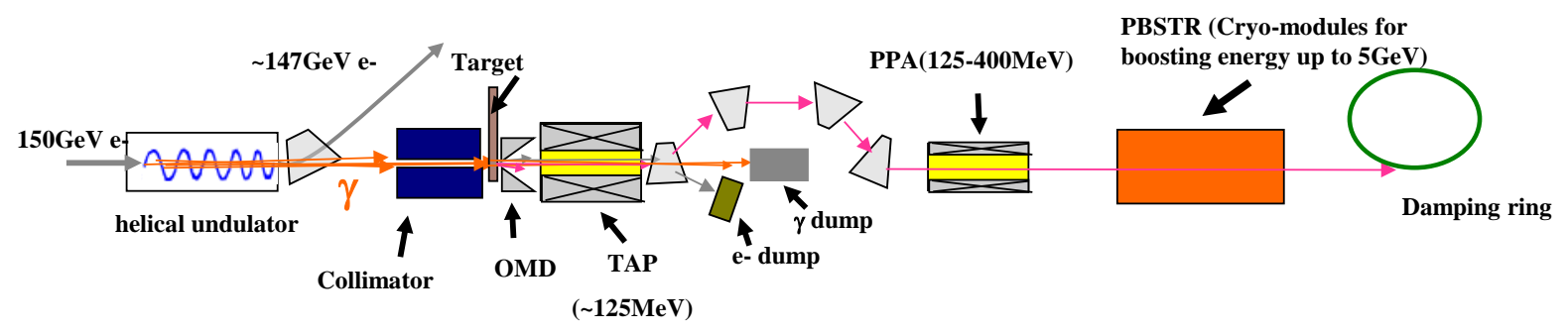

Figure 9b: Zoom on the ILC $\mathrm{e}^{+}$source

Another approach has been recently studied [41]. Instead of using compact targets, granular targets made of a large number of small spheres are considered. The idea is based on the high ratio between surface and volume of the spheres which makes easier the heat dissipation. Such idea is investigated for the ILC positron source [42].

\section{$7.2 \mathrm{LHeC} \mathrm{e}^{+}$sources}

A presentation of the $\mathrm{LHeC}$ project is given in [43]. The requested LHeC flux (a factor 300 compared to SLC - see Table 2) for the pulsed option at $140 \mathrm{GeV}$ could be obtained, in a first approximation, with $10 \mathrm{e}^{+}$ target stations working in parallel.

Figure 10 illustrates a possible option for the $140 \mathrm{GeV}$ case, where the repetition rate is $10 \mathrm{~Hz}$. The idea and the concept are developed in the CDR [44]. The consequence implies installing 2 RF deflectors upstream and same downstream. Experience exists, on the CLIC Test Facility (CTF3) for RF deflectors at $3 \mathrm{GHz}$ and 2 lines in parallel [45].

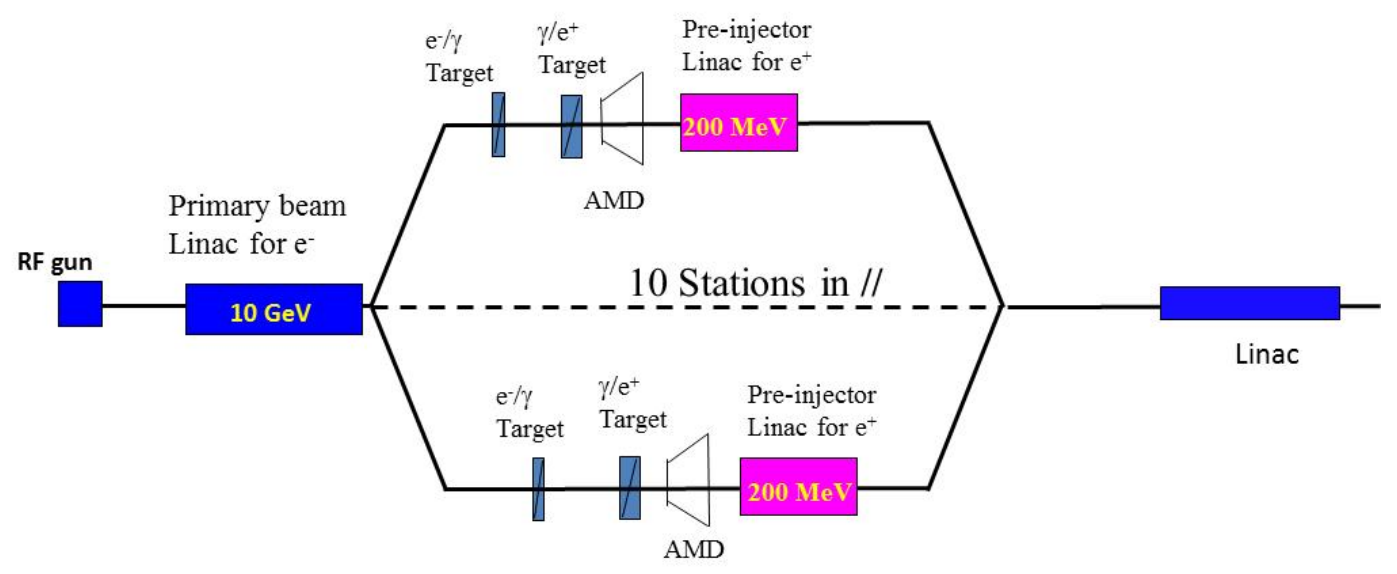

Figure 10: LHeC brilliant $\mathrm{e}^{+}$sources

The requested LHeC flux (a factor 7300 compared to SLC) for the CW option, needs a very important study and investigation.

\subsection{SuperKEKB $e^{+}$source}

In the SuperKEKB [46] injector linac, which is undergoing upgrade construction, positrons are produced by 3.2-GeV primary electrons of $10 \mathrm{nC}$ bunch intensity impinging on a tungsten target. Two bunches of positrons in $96 \mathrm{~ns}$ interval are generated in a beam pulse. An amorphous tungsten target of $14 \mathrm{~mm}$ thickness $\left(4.0 \chi_{0}\right)$ is used in a commissioning stage and it may be upgraded to a crystal tungsten target later to improve positron yield. The positrons from the target are captured by an AMD consisting of $5 \mathrm{~T}$ SLAC-type flux concentrator and $0.4 \mathrm{~T}$ DC solenoids. They are accelerated by six 2 m-long large aperture (diameter $30 \mathrm{~mm}$ ) S-band accelerating structures up to $120 \mathrm{MeV}$ in the solenoidal focusing region. The structure may be upgraded to L-band counterparts of $35 \mathrm{~mm}$ aperture. The $\mathrm{e}^{+}$are accelerated up to $1.1 \mathrm{GeV}$ and injected into a damping ring. After $40 \mathrm{~ms}$ of radiation damping, the low-emittance positrons are sent back to the linac and they are accelerated to $4.0 \mathrm{GeV}$ in downstream accelerator modules. The positron 
intensity for injection is $4 \mathrm{nC}$ per bunch. Figure 11 shows the general layout where the beam parameters are reported.

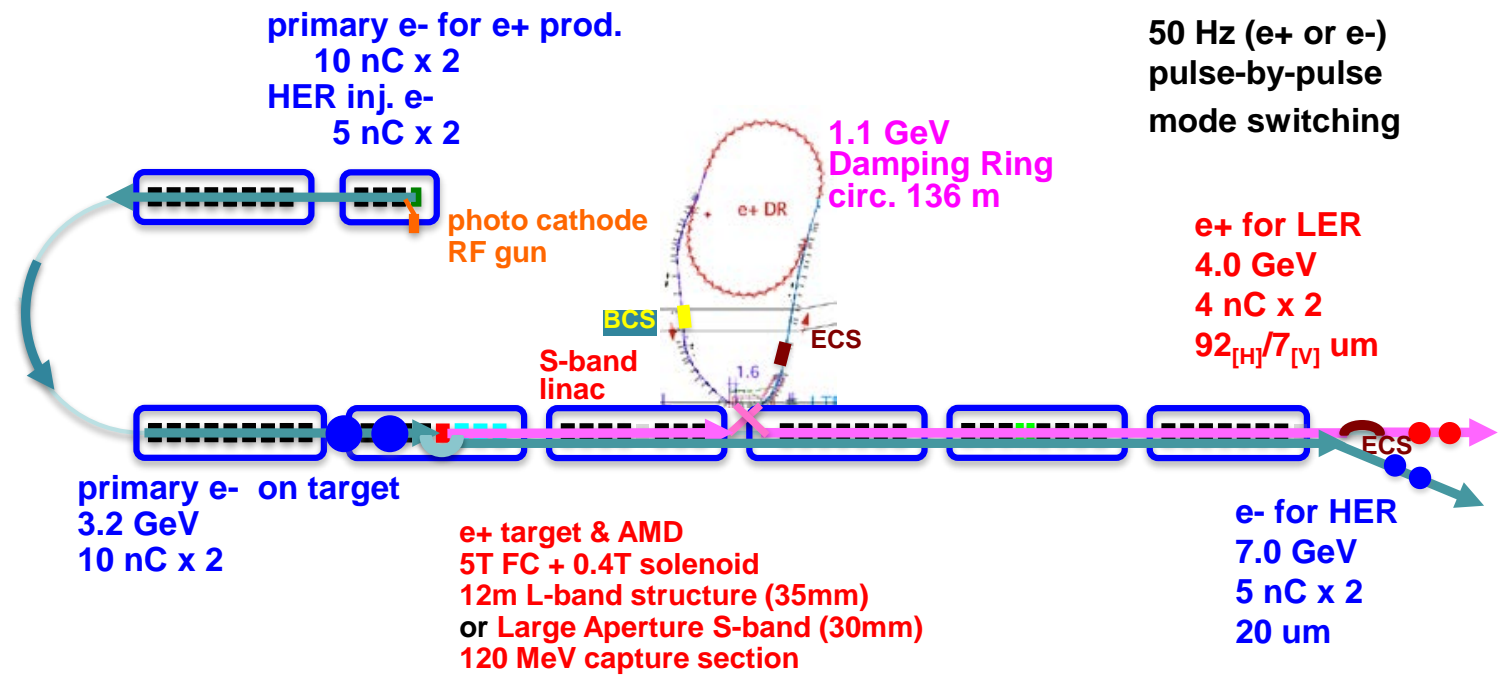

Figure 11: General layout of SuperKEKB

\subsection{SuperB $e^{+}$source}

In SuperB [47] the injection is at 30Hz with an injected charge per bunch of $\sim 300$ pC. Positrons are produced by electrons accelerated in a linac of $1.5 \mathrm{GeV}$, impinging on a positron converter target. Figure 12 shows a general layout. A capture section in $\mathrm{S}$ band but with larger iris provides the longitudinal post acceleration and bunching. Then another S-band linac is used to accelerate positrons up to $1 \mathrm{GeV}$ before DR injection where the longitudinal acceptance is $\sim+-1 \%$. Studies on the positron capture and acceleration system assure that the required positron yield is achieved with S-band linacs and good conversion efficiency. This solution has been preferred since it is well tested. An alternative solution, based on L-band capture and accelerating sections, showed better performances and could be adopted later if it is proven that can reduce the costs.

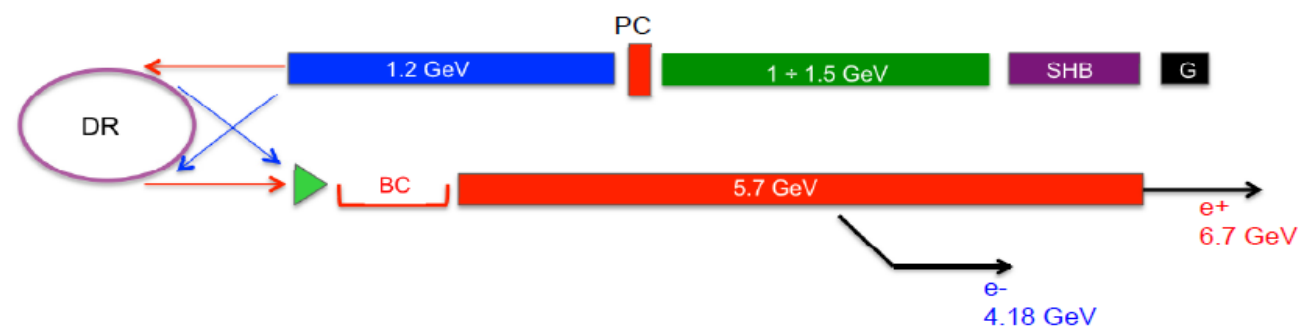

Figure 12: General layout of Super B

\section{Summary}

The CLIC history spans over 27 years with many changes, upgrades, failures and success.

The requested CLIC $\mathrm{e}^{+}$flux is very challenging (a factor 20 compared to what has been achieved on SLC). However the requested CLIC unpolarized $\mathrm{e}^{+}$source parameters seem reachable based on channeling process using a hybrid targets configuration. But experimental demonstrations are mandatory. For the CLIC polarized $\mathrm{e}^{+}$source, many studies have been performed and many paths explored, based on Compton rings, ERL and undulators. The Conceptual Design Report is just published. Studies related to the CLIC $\mathrm{e}^{+}$sources are on-going, in close collaboration with many institutes around the world.

Other projects, ILC, LHeC, SuperB's, involving brilliant positron sources are studied with a lot of exciting challenges. 


\section{References}

[1] G. Guignard (Ed.) et al., “A $3 \mathrm{TeV} \mathrm{e}^{+} \mathrm{e}^{-}$Linear Collider based on CLIC technology”, CERN 2000-008PS, July 2000

[2] LCWS08 and ILC08 workshop: November 2008 http://www.linearcollider.org/lcws08/

[3] J.D. Lawson, "Linear collider constraints: some implications for future accelerators”, CLIC Note 1

[4] S. Stapnes et al., "The CLIC Programme: Towards a Staged $\mathbf{e}^{+} \mathbf{e}^{-}$Linear Collider Exploring the Terascale: CLIC Conceptual Design Report”, CERN-2012-005; ANL-HEP-TR-12-51; KEK-Report-2012-2

[5] http://clic-study.org/

[6] F. Tecker (Ed.) et al, “CLIC 2008 parameters”, CLIC Note 764, October 2008

[7] J. Sheppard, “CLIC electron beam experiment”, presented at the LCWS'09 workshop, Albuquerque, September 2009.

[8] A. Ferrari, A. Latina, L. Rinolfi, " Design studies of the CLIC Injector and Booster Linacs with the 2007 beam parameters", CLIC Note 737, May 2008.

[9] F. Antoniou, Y. Papaphilippou, "Optics design considerations for the CLIC Pre-Damping Rings" presented at EPAC08, Genoa, Italy, May 2008.

[10] F. Stulle, L. Rinolfi, D. Schulte, A. Latina, A. Ferrari, "CLIC Main beam dynamics in the ring to main LINAC transport”, CERN-ATS-2009-090 - CLIC-Note-790, presented at PAC2009, Vancouver, Canada, May 2009

[11] J.P. Delahaye, “An injector complex for the main beams of the CERN linear collider”, CLIC Note 230, presented at EPAC 94, London, UK, June 1994

[12] G. Lemeur, LAL, private communication

[13] L. Rinolfi, "A CLIC Injector Complex for the Main Beams", CERN-OPEN-98-010, CLIC Note 354

[14] T. Kamitani, L. Rinolfi, "Positron production at CLIC", CLIC note 465, March 2001

[15] R.Chehab "Positron Sources” CAS, CERN-94-01, Vol. II

[16] L. Rinolfi, T. Kamitani, "The CLIC positron production scheme", CLIC Note 537, in XXI ${ }^{\text {st }}$ Linear Accelerator Conference, Gyeongju, Korea, August 2002

[17] E. Eroglu, Uludag University, Turkey, private communication

[18] R.Chehab et al. "Study of a positron source generated by photons from ultrarelativistic channelled particles” presented at PAC, Chicago, 1989

[19] R. Chehab et al., "Radiation damage study of a monocrystalline tungsten positron converter”, CERN/PS 98-17(LP), CLIC Note 369, LAL/RT 98-02, presented at EPAC98, Stockholm, June 1998

[20] R.Chehab et al. "Experimental study of a crystal positron source” Physics Letters B 525 (2002) 41-48

[21] X.Artru et al. "Summary of experimental studies at CERN on a positron source using crystal effects" Nuclear Instruments and Methods B 240 (2005) 762-776

[22] X.Artru et al. "Polarized and unpolarized positron sources for linear colliders” NIM B 266 (2008)

[23] O. Dadoun, I. Chaikovska, R. Chehab, F. Poirier, L. Rinolfi, V. Strakhovenko, A. Variola, A. Vivoli, "Study of a hybrid positron source using channeling for CLIC", CLIC Note 808

[24] A. Vivoli, I. Chaikovska, R. Chehab, O. Dadoun, P. Lepercq, F. Poirier, L. Rinolfi, V. Strakhovenko, A. Variola, "The CLIC Positron Capture and Acceleration in the Injector Linac", CLIC Note 819

[25] F. Poirier, O. Dadoun, P. Lepercq, L. Rinolfi, A. Variola, A. Vivoli, "Dynamics on the positron capture and accelerating sections of CLIC", CERN-OPEN-2011-034, CLIC-Note-877

[26] O. Dadoun, I. Chaikovska, R. Chehab, P. Lepercq, F. Poirier, L. Rinolfi, V. Strakhovenko, A. Variola, A. Vivoli, C. Xu, "The baseline positron production and capture scheme for CLIC", CLIC Note 824, presented at IPC10, Kyoto, Japan, May 2010

[27] T. Takahashi, "Status of Hybrid target R\&D at KEK-Linac”, POSIPOL12 workshop, DESY Zeuthen, Berlin, Germany, September 2012

[28] F. Zimmermann et al., "CLIC polarized positron source based on laser Compton scattering”, CERNAB-2006-073, CARE-ELAN-DOCUMENT-2006-006, CLIC-Note-674, presented at EPAC06, Edinburgh, UK, June 2006

[29] L. Rinolfi et al. "The CLIC positron source based on Compton schemes”, CLIC Note 788, presented at PAC09, Vancouver, Canada, May 2009

[30] L. Rinolfi et al., "The CLIC electron and positron polarized sources”, CERN-OPEN-2010-017, CLIC Note 817, presented at XIII ${ }^{\text {th }}$ International workshop on polarized sources, targets \& polarimetry (PST 2009), Ferrara, Italy, September 2009

[31] E. Bulyak, P. Gladkikh, T. Omori, L. Rinolfi, J. Urakawa, "Beam Dynamics in Compton storage rings with laser cooling”, CERN-ATS-2010-121, CLIC-Note-855, presented at IPC10, Kyoto, Japan, May 2010 
[32] P. Gladkikh et al., "3.5 GeV Superconducting Positron Stacking Ring”, POSIPOL12 workshop, DESY Zeuthen, Berlin, Germany, September 2012

[33] T. Omori, “ERL based Compton scheme \& requirements to lasers”, POSIPOL 2007, Orsay, May 2007

[34] T. Omori, L. Rinolfi, “ERL Compton scheme for CLIC”, CLIC09 workshop, CERN, October 2009

[35] V. Yakimenko, "Compton linac for polarized positrons" CLIC09 workshop, CERN, October 2009

[36] W. Gai, "Update on Undulator based positron source for CLIC”, CLIC09 workshop, CERN, Oct. 2009

[37] W. Liu, W. Gai, L. Rinolfi, J. Sheppard, "An undulator based polarized positron source for CLIC”, CLIC Note 856, presented at IPAC 10, Kyoto, Japan, May 2010

[38] International Linear Collider Reference design Report (http://www.linearcollider.org/rdr) August 2007

[39] A. Ushakov and al., "Polarized positrons for the ILC, Update on simulations”, DESY-12-018

[40] G.Alexander et al. "Undulator-based production of polarized positrons, NIM A 610 (2009)

[41] P. Sievers, "Granular vs compact positron converters: advantages \& applications to hybrid sources", POSIPOL12 workshop, DESY Zeuthen, Berlin, Germany, September 2012

[42] C. Xu, PhD thesis at IHEP/Beijing, May 2012

[43] F. Zimmermann et al., "Positron options for the Linac-Ring LHeC", CERN-ATS-2012-135, presented at IPAC’12, New-Orleans, USA, May 2012

[44] LHeC study group, "A large hadron electron collider at CERN”, Journal of Physics G, Volume 39, Number 7, July 2012.

[45] R. Corsini, A. Ferrari, L. Rinolfi, P. Royer, F. Tecker, "Experimental results on electron beam combination and frequency multiplication", Physical Review Special Topics - Accelerators and Beams 7 (2004) 040101, CERN-AB-2005-018, CLIC-Note-624

[46] T. Kamitani, "Positron Injector Linac Upgrade for SuperKEKB", presented at Linear Accelerator Conference 2012, Tel-Aviv, Israel, September 2012

[47] S. Guiducci et al., "Baseline Design of the Superb Factory Injection System", presented at IPAC'12, New Orleans, US, May 2012. 